\title{
Determination of Maximum Optical Range of FSO Link Affected by Atmospheric Attenuation in Spatial Diversity
}

\author{
Poonam Agrawal $^{1}$, Sandeep Kumar Toshniwal ${ }^{2}$ \\ ${ }^{1}$ P.G. Scholar, ${ }^{2}$ Associate Professor (Electronics \& Communication) \\ Kautilya Institute of Technology \& Engineering, Jaipur, Rajasthan, India
}

\begin{abstract}
In this paper we have analyzed free space optical communication system affected by atmospheric attenuation in spatial diversity. Spatial diversity means different numbers of transmitters and receivers in FSO link. We have taken five configurations of FSO which are: $1 \times 1,2 \times 2,4 \times 4,8 \times 8$, and $16 \times 16.2 \times 2$ configuration means there are 2 transmitters and 2 receivers in FSO link. FSO link is analyzed at 1550 $\mathrm{nm}$ wavelength for different values of input power. Attenuation is taken as clear $(0.43 \mathrm{~dB} / \mathrm{Km})$, haze $(4.3$ $\mathrm{dB} / \mathrm{Km})$ and fog $(43 \mathrm{~dB} / \mathrm{Km})$. Maximum optical range of FSO link is calculated for constant Q factor of value 5.17. Eye diagrams are generated to produce the desired results.
\end{abstract}

Keywords: FSO, Attenuation, Spatial Diversity, Bit Error Rate, Q Factor

\section{INTRODUCTION}

The field of wireless communication has been broadly investigated in order to effort the advantages it has over connected networks such as mobility and flexibility. The requirements for bandwidth on wireless communication systems today is accelerate at an exponential rate. Many bandwidth exacting applications (multimedia) are being formed these days. Thus conventional voice communication is not the only demand of wireless communication in today's network. The main challenge is to design more adaptive and ascendible systems that can supply high data rates to support the increasing demand for bandwidth. In view of this, many wireless systems have been developed to address the demand for high information carrying capacity.

The sum of data that can be conveyed in any communication system is directly connected to the bandwidth of the carrier which is directly attached to the carrier frequency. Optical signals use a frequency range of $20 \mathrm{THz}-375 \mathrm{THz}$ and could therefore guarantee very high data rates. Optical communication systems thus promise the highest possible content carrying capacity. The theoretical content carrying capacity of free space optical communication systems over stages that of microwave systems. The information carrying capability of microwave systems is the highest so far amongst the available wireless networks. Free Space Optical (FSO) communication is the causing of high speed data over long distances using optical signals through free space.

Free Space Optical (FSO) communication can be reasoned as in sight technology for future generation communication due to its wide range of applications. Some of its applications are links affecting satellites (inter-satellite communication), High Altitude Platforms (HAPs), Unmanned Aerial Vehicles (UAVs), terrestrial communications, aircraft and shipto-ship communication. FSO can provide high data rates in areas where it is hard or unfeasible to lay optical fiber cables. Again, it can be used in both military and civilian applications.

FSO communication process is the transmission of data over large areas using modulated optical signals via free space (or an unguided transmission media). The unguided transmission media could be space, water, atmosphere or a mixture of any of these media. Since this research defines terrestrial transmissions, the medium of interest is the atmosphere. The information to be transmitted can be modulated in its phase, intensity or frequency of the optical wave. An FSO link is totally a line-of-sight (LOS) technology. 


\section{FEATURES}

The primary features of FSO technology are mentioned below:

$>$ Large Bandwidth: The amount of data that can be transmitted in any communication channel is directly proportional to the bandwidth of the carrier which is directly proportional to the carrier frequency. The permissible data bandwidth may be up to $20 \%$ of the carrier frequency. Optical waves use a frequency range of $20 \mathrm{THz}-375 \mathrm{THz}$ and would therefore guarantee very large bandwidth or data rate. This is due to the electromagnetic spectrum, the optical carrier frequency which consists of visible, ultraviolet and infrared light are far larger than the radio frequency.

> Narrow Beam width: The beam width of optical waves is very narrow. Common laser beams include diffraction limited divergence angle between $0.01-0.10 \mathrm{mrad}$. This states that the optical power is confined within a narrow area and therefore needs a line-of-sight (LOS) between transmitter and receiver. Optical waves are protective to electromagnetic interference and give opportunity for unlimited frequency reuse because of this characteristic.

$>$ Highly Secured: Optical waves are highly secured with low possibility of interception and low possibility of detection (LPI/LPD) characteristics. Laser beam produced by FSO systems is narrow and invisible. This makes them tough to capture and even more tough to crack. Optical waves cannot be detected with spectrum analyzers or RF meters.

> Weather dependency: FSO execution highly depends on the patterns of weather in the installation area because atmospheric situations like fog, rainfall, temperature, dust particles, atmospheric turbulence and smoke directly influence the availability and reliability of the FSO link.

> Unlicensed Spectrum: RF signals face a major challenge of interference due to congestion of the RF spectrum. Local regulatory bodies like Office of Communication in the United Kingdom, National Communications Authority (NCA) in Ghana, Federal Communication Commission in the United States and Department of Telecommunications in India observe the use of the RF spectrum in their concerned countries. To use RF spectrum needs license from the local bodies which cost a lot of money. The utilization of the electromagnetic spectrum for FSO does not need any form of spectrum license from local bodies and therefore is a major cost benefit over the use of RF spectrum.

Easy installation: The time it consumes for FSO link to become functional from its beginning of installation to its alignment is relatively small. The major requirement is to assure direct LOS without any kind of obstruction between the transmitter and receiver ends. This is not similar to the use of fiber optic cables which needs right of way and trenching additional extra cost to the installation work.

\section{PROPOSED DESIGNS}

We have used different configurations of FSO link and calculated range for each configuration keeping $\mathrm{Q}$ factor constant at 5.17. Different configurations are shown below:

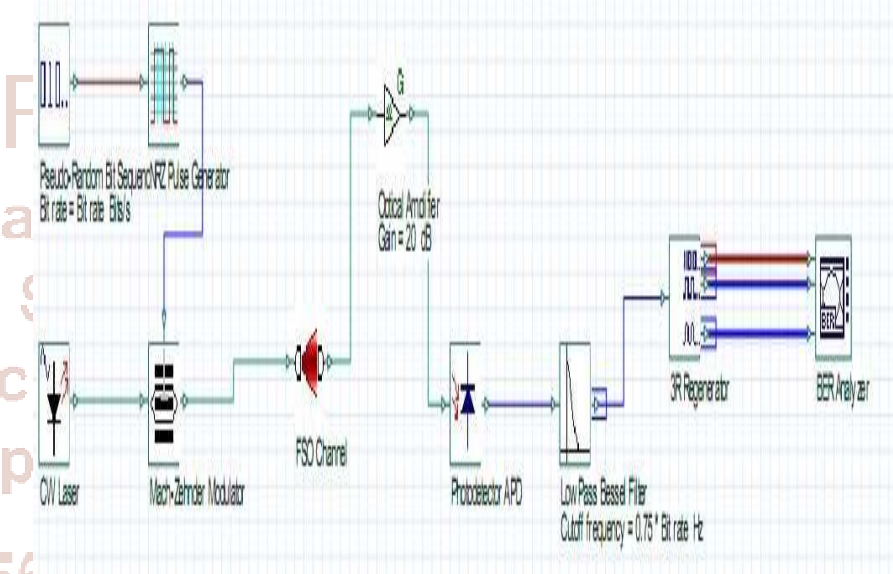

Fig-4: FSO system (1x1)

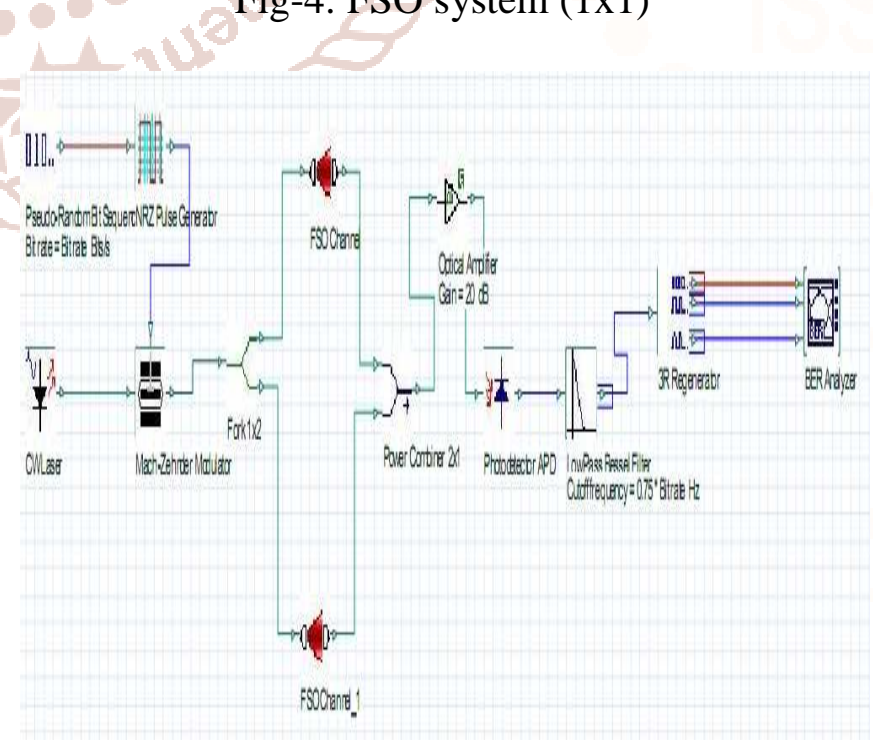

Fig-5: FSO system (2x2) 
International Journal of Trend in Scientific Research and Development (IJTSRD) ISSN: 2456-6470

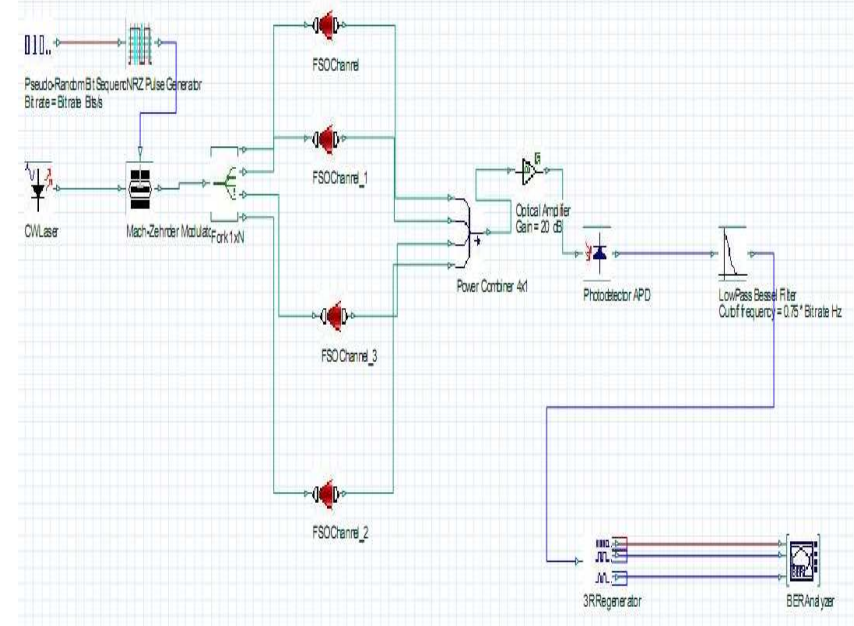

Similarly $16 \times 16$ architecture is also designed to perform the analysis.

\section{SIMULATION ANALYSIS}

All FSO systems are simulated to determine the range possible for different input power at different atmospheric conditions for $1550 \mathrm{~nm}$ frequency. In this analysis value of $\mathrm{Q}$ factor is maintained constant at 5.17 and minimum BER is $10^{-7}$. Results for different power under different climatic conditions are shown below:

Table 1 Simulation results for different power at clear climate

Fig-6: FSO system (4x4)

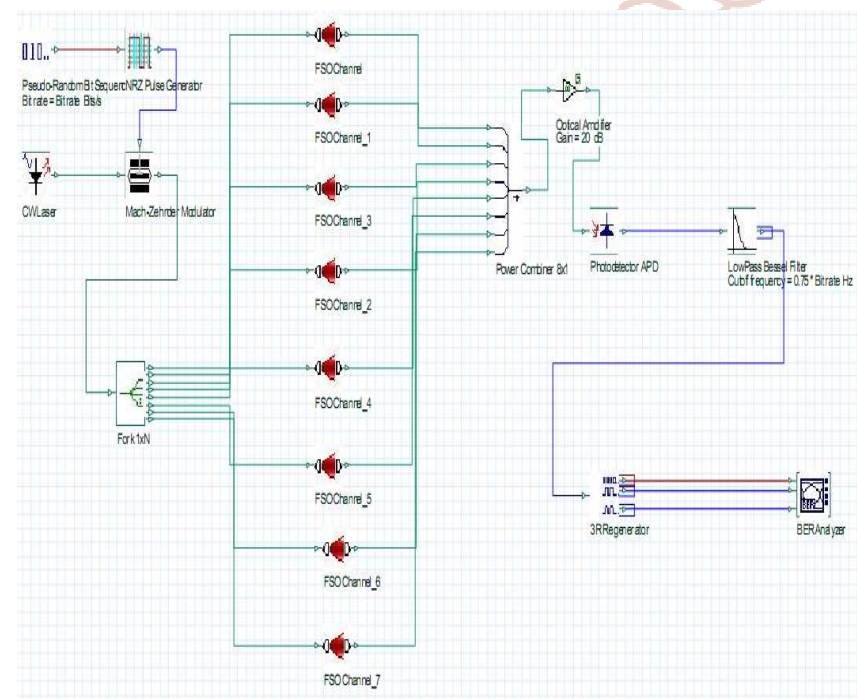

\begin{tabular}{|c|c|c|c|c|}
\hline \multicolumn{5}{|c|}{$\begin{array}{c}\text { Attenuation }=0.43 \mathrm{~dB} / \mathrm{Km}, \text { Q-Factor } \\
\text { and }\end{array}$ Minimum BER $=10^{-7}$} \\
\hline \multirow{2}{*}{$\begin{array}{c}\text { Maximum Possible Range } \\
(\mathrm{Km})\end{array}$} \\
\cline { 2 - 5 } & $\begin{array}{c}10 \\
\mathrm{dBm}\end{array}$ & $\begin{array}{c}15 \\
\mathrm{dBm}\end{array}$ & $\begin{array}{c}20 \\
\mathrm{dBm}\end{array}$ & $\begin{array}{c}25 \\
\mathrm{dBm}\end{array}$ \\
\hline $1 \times 1$ & 38.04 & 45.88 & 53.95 & 63.61 \\
\hline $2 \times 2$ & 43.06 & 50.82 & 59.31 & 68.16 \\
\hline $4 \times 4$ & 47.78 & 56.05 & 62.87 & 73.75 \\
\hline $8 \times 8$ & 52.18 & 61.13 & 68.21 & 80.04 \\
\hline $16 \times 16$ & 57.45 & 66.54 & 75.26 & 84.80 \\
\hline
\end{tabular}

Fig-7: FSO system (8x8)

Table 2 Simulation results for different power at haze climate

\begin{tabular}{|c|c|c|c|c|}
\hline \multirow{2}{*}{ Attenuation $=4.3 \mathrm{~dB} / \mathrm{Km}$, Q-Factor $=\mathbf{5 . 1 7}$ and Minimum BER $=\mathbf{1 0}^{-7}$} \\
\hline \multirow{2}{*}{ Configuration } & \multicolumn{4}{|c|}{ Maximum Possible Range $(\mathrm{Km})$} \\
\cline { 2 - 5 } & $\mathbf{1 0} \mathbf{d B m}$ & $\mathbf{1 5} \mathbf{~ d B m}$ & $\mathbf{2 0} \mathrm{dBm}$ & $\mathbf{2 5} \mathbf{~ d B m}$ \\
\hline $1 \times 1$ & 7.133 & 8.021 & 8.911 & 9.941 \\
\hline $2 \times 2$ & 7.705 & 8.573 & 9.621 & 10.541 \\
\hline $4 \times 4$ & 8.162 & 9.059 & 10.206 & 11.255 \\
\hline $8 \times 8$ & 8.953 & 9.805 & 10.662 & 11.814 \\
\hline $16 \times 16$ & 9.455 & 10.429 & 11.310 & 12.439 \\
\hline
\end{tabular}

Table 3 Simulation results for different power at fog climate

\begin{tabular}{|c|c|c|c|c|}
\hline Attenuation $=43$ & $\mathrm{Km}, \mathrm{Q}-\mathrm{F}$ & $r=5.17$ & Minimu & $E R=10$ \\
\hline Confiouretion & & mum Pos & le Range & \\
\hline Cominguration & $10 \mathrm{dBm}$ & $15 \mathrm{dBm}$ & $20 \mathrm{dBm}$ & $25 \mathrm{dBm}$ \\
\hline $1 \mathrm{x} 1$ & 1.007 & 1.180 & 1.293 & 1.391 \\
\hline $2 \times 2$ & 1.145 & 1.253 & 1.351 & 1.452 \\
\hline $4 \times 4$ & 1.206 & 1.301 & 1.395 & 1.512 \\
\hline $8 \times 8$ & 1.266 & 1.376 & 1.458 & 1.567 \\
\hline $16 \times 16$ & 1.319 & 1.436 & 1.528 & 1.632 \\
\hline
\end{tabular}


Graphical representation of Table 1, 2 and 3 are shown below:

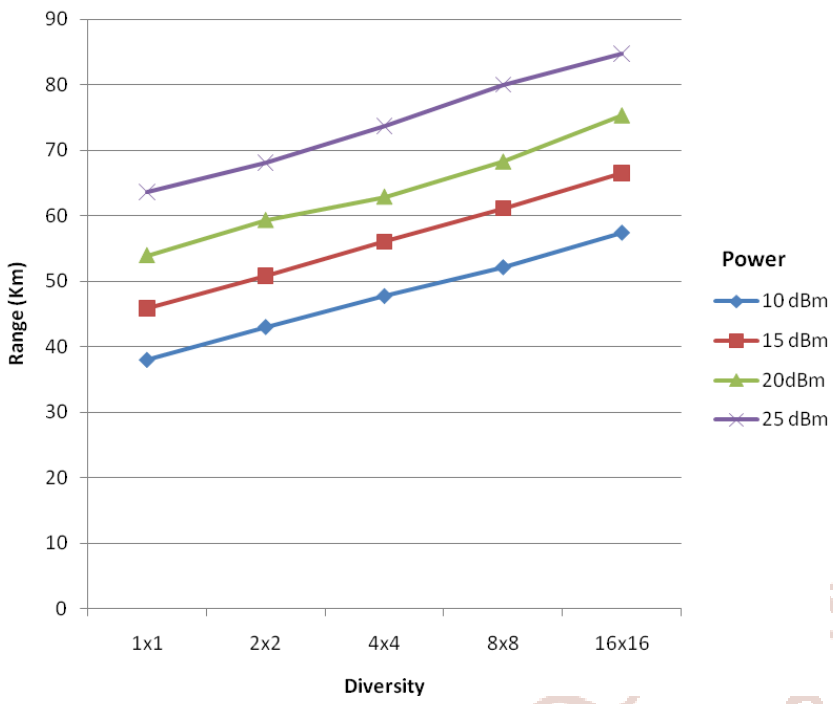

Fig. 8 Comparison graph of range for different power at clear climate

From Fig. 8 we can conclude that as we increase the input power, optical range increases with increasing diversity. Range is above $80 \mathrm{Km}$ for clear climate where attenuation is equal to $0.43 \mathrm{~dB} / \mathrm{Km}$. Similarly for other attenuation values comparison graphs are shown in Fig. 9 \& 10.

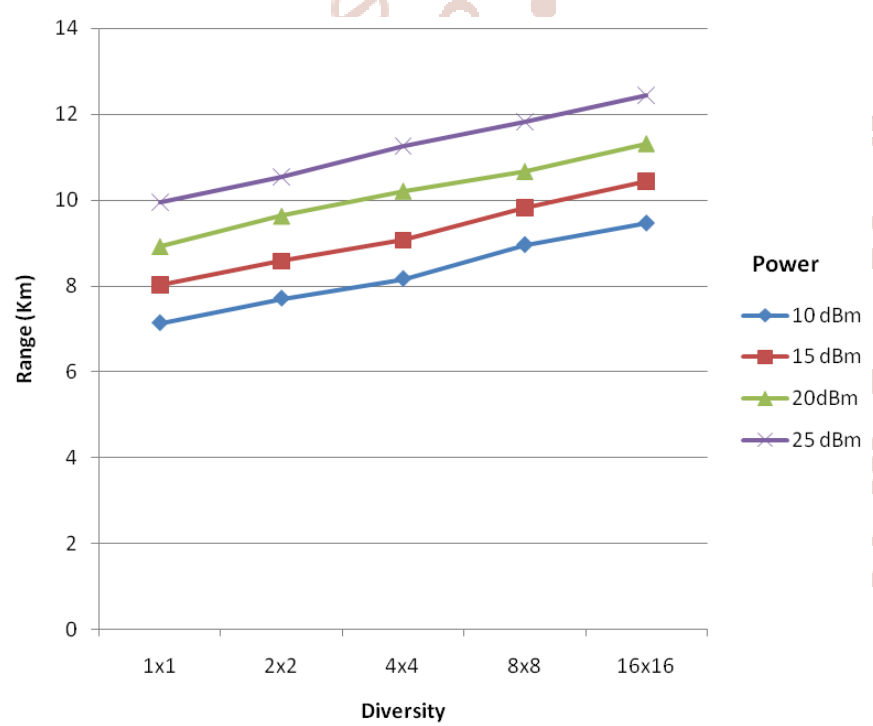

Fig. 9 Comparison graph of range for different power at haze climate

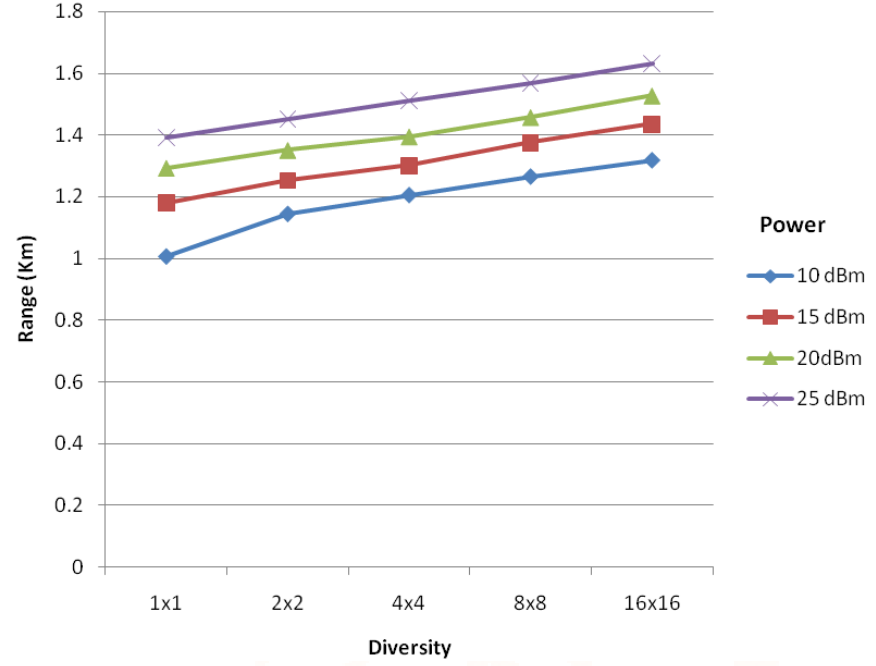

Fig. 10 Comparison graph of range for different power at fog climate

As we increase the attenuation value range decreases as compared to other climatic conditions.

\section{EYE DIAGRAM RESULTS}

In this section, eye diagrams for different values of attenuation at $10 \mathrm{dBm}$ powers under different climatic conditions for $1 \times 1$ type of configuration are shown.

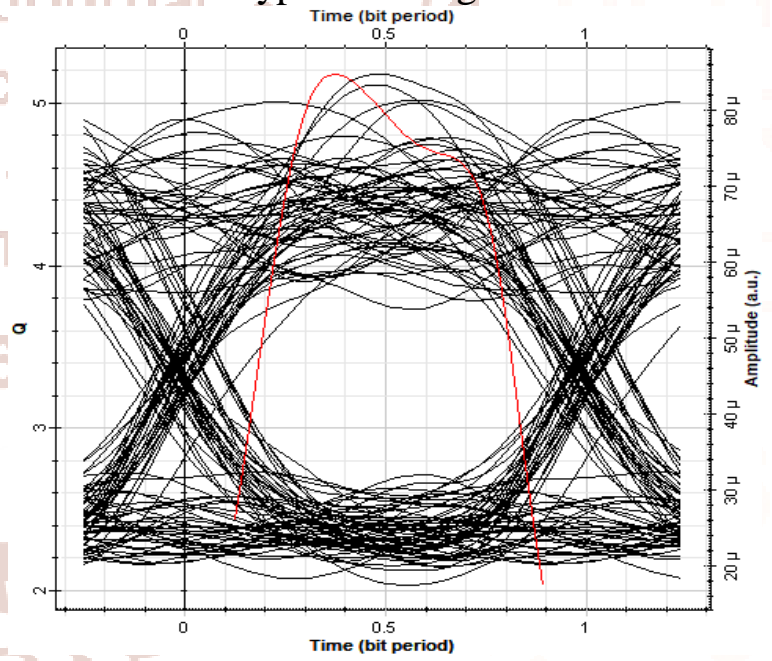

Fig 11 Eye diagram for 1x1 FSO at clear climate

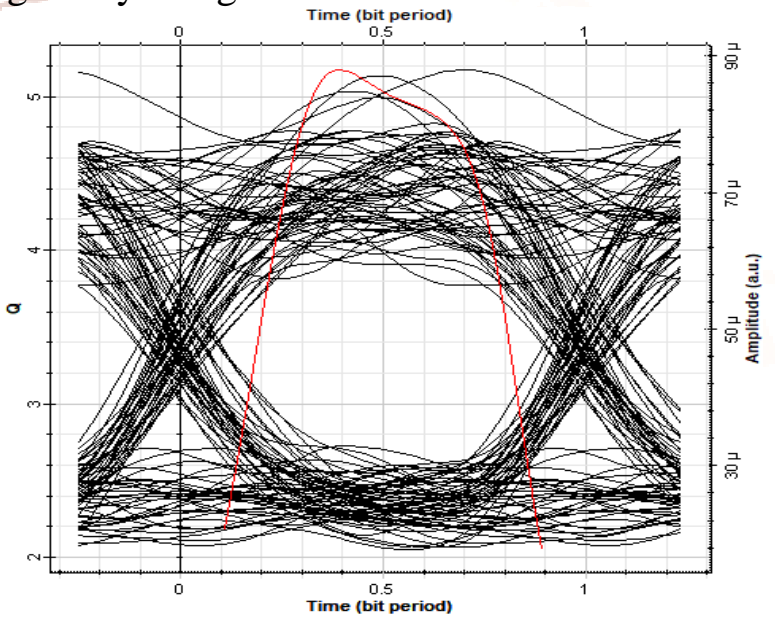

Fig 12 Eye diagram for 1x1 FSO at haze climate 


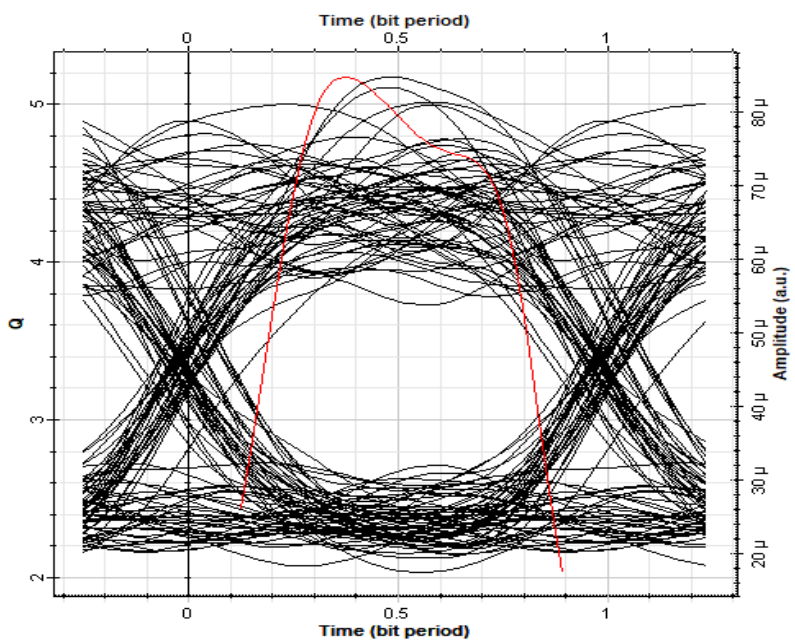

Fig 13 Eye diagram for 1x1 FSO at fog climate

\section{CONCLUSIONS}

FSO provides several benefits over previously developed methods in optical, radio or microwave domain. Less installation cost and less installation time are the main benefits of FSO system. In FSO system atmospheric conditions such as haze, fog and so on is the main factor which causes distortion in optical signal. We have successfully analyzed different configurations of FSO system and calculated range at different power under the influence of different climatic conditions.

\section{REFERENCES}

1. S. Vigneshwaran, I.Muthumani, and A. S. Raja, "Investigations on free space optics communication system," International Conference on Information Communication \& Embedded Systems, IEEE, 2013.

2. J. Singh and N. Kumar, "Performance analysis of diferent modulation format on free space optical communication system," Optik, 2013.
3. S. A. Al-Gailani, A. B. Mohammad, and R. Q. Shaddad, "Enhancement of free space optical link in heavy rain attenuation using multiple beam concept," Optik,vol.124,no.21, 2013.

4. Z. Ghassemlooy, J. Perez, and E. Leitgeb, "On the performance of FSO communications links under sandstorm conditions," International Conference on Telecommunications, IEEE, June 2013.

5. K. Rammprasath and S. Prince, "Analyzing the cloud attenuation on the performance of free space optical communication," International Conference on Communication and Signal Processing, 2013.

6. S. A. Al-Gailani, A. B. Mohammad, R. Q. Shaddad, and M. Y. Jamaludin, "Single and multiple transceiver simulation modules for freespace optical channel in tropical malaysian weather," IEEE Business Engineering and Industrial Applications Colloquium, April 2013.

7. H. A.Willebrand and B.S.Ghuman, "Fiber optics without fiber," IEEE Spectrum, 2001.

8. V. Sharma and G. Kaur, "High speed, long reach OFDM-FSO transmission link incorporating OSSB and OTSB schemes," Optik, 2013.

9. R. K. Z. Sahbudin, M. Kamarulzaman, S. Hitam, M. Mokhtar, and S.B.A.Anas, "Performance of SAC OCDMA-FSO communication systems," Optik, 2013.

10. Manisha Prajapat and Chetan Selwal, "Free Space Optical Link Performance Simulation under Different Atmospheric Conditions and Diversity", IEEE, 2017. 